einen mRS-Wert von 0-3 Punkten, hingegen nur $30 \%$ der $\mathrm{Pa}$ tienten ohne OAK. Die Sterberate war mit OAK deutlich geringer $(\mathrm{OR}=0,29$ für nicht lobäre und 0,26 für lobäre Blutungen). Schlaganfälle jeglicher Art traten mit OAK nur etwa halb so oft auf, lediglich erneute ICH wurden unter OAK etwas, aber nicht signifikant häufiger als ohne beobachtet $(\mathrm{OR}=1,12$ bei nicht lobären und 1,26 bei lobären Blutungen). Signifikant seltener (-60\%) kam es dagegen zu ischämischen Schlaganfällen.

Die Wiederaufnahme der OAK nach einer überstandenen Hirnblutung geht folglich mit einer drastisch reduzierten Mortalität und einem deutlich verbesserten funktionellen Ergebnis nach einem Jahr einher, folgerte Biffi, und zwar unabhängig vom $\mathrm{CHAD}_{\mathrm{S}}$ - und HAS-BLED-Score.
Daniel Hanley, Johns-Hopkins-Universität Baltimore, gab zu bedenken, dass nur relativ wenige Patienten erneut eine OAK bekamen und nur wenige Schlaganfälle und erneute Hirnblutungen innerhalb eines Jahres auftraten. „Ein Jahr ist hier doch eine recht kurze Zeit“, so Hanley. Zudem könnten trotz PropensityScore-Matching eher die Patienten mit einer besseren Prognose erneut eine OAK erhalten haben, was die Resultate deutlich zugunsten der OAK verzerrt haben könnte. Hier wären also weitaus größere und länger dauernde Studien nötig.

Thomas Müller, Springer Medizin

Contemporary Clinical Issue Plenary Session, 24. 4. 2017.

69. AAN, Boston, 22. - 28.4.2017

\title{
Intensive Blutdrucksenkung bei Hirnblutung doch von Nutzen?
}

\begin{abstract}
Eine intensive systolische Blutdrucksenkung unter
$140 \mathrm{mmHg}$ bei einer Hirnblutung ergab in der

ATACH-2-Studie keine Vorteile. Nach Daten einer

erneuten Analyse scheinen aber Patienten mit an-

fänglich großem Hämatomvolumen zu profitieren.
\end{abstract}

Bei einer Hirnblutung soll der systolische Druck durch eine antihypertensive Therapie rasch unter $180 \mathrm{mmHg}$ gesenkt werden, um eine Expansion des Hämatomvolumens zu vermeiden. $\mathrm{Ob}$ eine noch stärkere Blutdrucksenkung mehr bringt, ist umstritten. Weder in der 2013 veröffentlichten Interventionsstudie INTERACT2 mit mehr als 2.800 Teilnehmern noch in der Studie ATACH-2, publiziert in 2016, gab es einen signifikanten Vorteil für Patienten mit einer intensiven antihypertensiven Therapie. Sterberate und funktionelles Ergebnis unterschieden sich in den Gruppen mit einem Therapieziel von $<180 \mathrm{mmHg}$ und $<140 \mathrm{mmHg}$ nicht wesentlich. Nun deutet eine Subgruppenanalyse der ATACH-2-Studie für einige Patienten doch auf einen gewissen Nutzen, so Dr. Adnan Qureshi, Universität Minneapolis: Liegt das anfängliche Hämatomvolumen über $10 \mathrm{~cm}^{3}$, scheint die intensivere Therapie eine weitere Hämatomexpansion besser zu vermeiden und die Sterberate etwas zu drücken.

\section{Geringere Hämatomexpansion, ...}

Zur Erinnerung: An ATACH-2 nahmen bis zum vorzeitigen Abbruch 960 Patienten mit intrazerebralen Blutungen teil. Sie waren in zwei Gruppen aufgeteilt worden: In der ersten wurde ein systolischer Wert unter $180 \mathrm{mmHg}$ angestrebt (Standardtherapie), in der zweiten Gruppe sollten es weniger als $140 \mathrm{mmHg}$ sein, die es innerhalb von zwei Stunden zu erreichen galt (intensivierte Therapie). Vorgeschrieben war ein Versuch mit Nicardipin bis zu einer Dosis von maximal $15 \mathrm{mg} / \mathrm{h}$ i.v.; falls dies nicht genügte, konnten die Studienärzte weitere Antihypertensiva wie Labetalol, Diltiazem oder Urapidil verwenden. Die Teilnehmer mussten zu Beginn einen systolischen Druck von mehr als $180 \mathrm{mmHg}$, ein anfängliches Hämatomvolumen von weniger als $60 \mathrm{~cm}^{3}$ sowie einen Wert auf der Glasgow Coma Scale von mindestens 5 Punkten aufweisen. Das Alter lag im Schnitt bei 62 Jahren, der initial gemessene systolische Blutdruck bei $200 \mathrm{mmHg}$.
Insgesamt verlief die Drucksenkung recht erfolgreich: Mit der Standardtherapie wurde im Schnitt ein Wert von $141 \mathrm{mmHg}$ erreicht, mit der intensivierten sank der systolische Druck im Mittel auf $129 \mathrm{mmHg}$. Letztlich lagen die Werte aber nicht weit auseinander, was sich deutlich auf den primären Endpunkt der Studie ausgewirkt haben dürfte: Nach drei Monaten waren in beiden Gruppen jeweils 38\% der Patienten tot oder schwer behindert (mRS-Wert von 4-6 Punkten). Ein gewisser Vorteil deutete sich zumindest bei der Hämatomexpansion an: Eine solche wurde bei $19 \%$ mit intensivierter und bei $24 \%$ mit Standardtherapie beobachtet, doch auch hier war der Unterschied nicht signifikant.

\section{... bessere Prognose}

Werden hingegen nur Patienten mit einem initialen Hämatomvolumen über $10 \mathrm{~cm}^{3}$ betrachtet, ergibt sich ein leicht anderes Bild. Eine Expansion des Hämatomvolumens um mehr als ein Drittel fanden die Ärzte um Qureshi hier bei 19\% mit intensiver und bei $28 \%$ mit konventioneller Blutdrucksenkung - der relative Unterschied von 33 \% lag knapp am Signifikanzniveau.

Kommt es zu einer relevanten Ausweitung der Blutung, ist die Prognose jedenfalls sehr ungünstig: In der Studie waren 52\% dieser Patienten nach drei Monaten tot oder schwer behindert, ohne Expansion hingegen nur 33\% - ein signifikanter Unterschied. Qureshi sieht daher durchaus einen gewissen Nutzen der intensiven Drucksenkung für Patienten mit initial großem Hämatomvolumen. Allerdings müsste es gelingen, die Expansion der Blutung bei deutlich mehr Patienten zu unterbinden. Da sich die erreichten Blutdruckwerte in den beiden Gruppen kaum unterschieden, bleibt aber die Frage nach einem Nutzen der intensiveren Drucksenkung offen. Augenscheinlich ist die Standardtherapie bereits so gut, dass eine intensivere Drucksenkung nicht nötig ist. Oder in anderen Worten: Eine starke Blutdrucksenkung scheint die Hämatomexpansion tatsächlich signifikant zu bremsen, dafür genügt aber meist die Standardtherapie. Lediglich bei initial großem Hämatomvolumen sollten Ärzte nach diesen Daten den Blutdruck noch stärker kontrollieren, als dies bislang der Fall ist. Thomas Müller, Springer Medizin

Clinical Trial Plenary Session, 25. 4. 2017. 69. AAN, Boston, 22. - 28.4.2017 